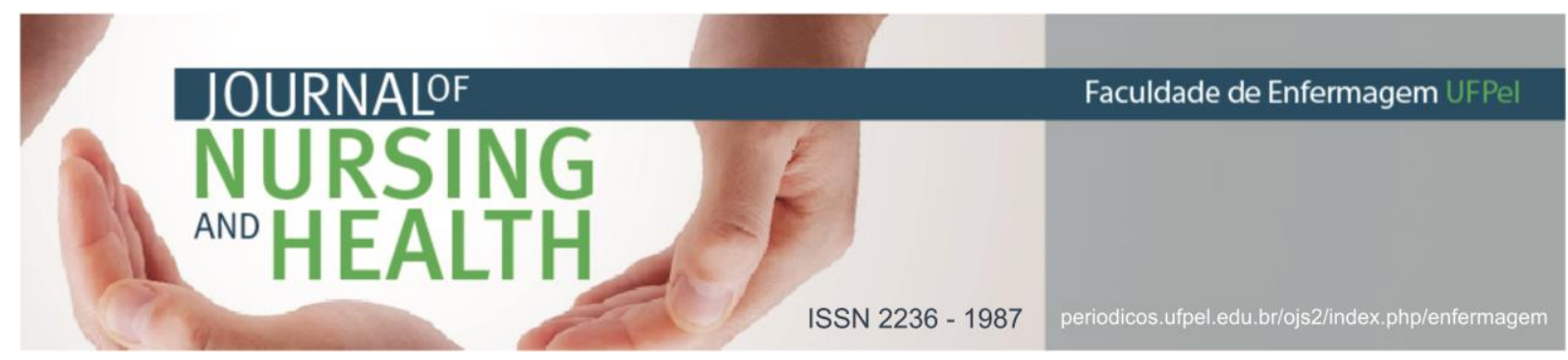

ARTIGO DE REVISÃO

\title{
Produção científica acerca do descolamento prematuro da placenta
}

\author{
Scientific production on abruptio placentae
}

\section{Producción científica sobre desprendimiento de la placenta}

\author{
Rocha, Bruna Dedavid ${ }^{1}$; Menezes, Francislene Lopes²; Zamberlan, Cláudia ${ }^{3}$; Gomes, Iris Elizabete
} Messa ${ }^{4}$; Bordignon, Juliana Silveira ${ }^{5}$

\begin{abstract}
RESUMO
Objetivo: analisar as produções científicas disponíveis na literatura, sobre descolamento prematuro da placenta (DPP). Métodos: trata-se de uma revisão integrativa de literatura. A busca foi realizada nas bases de dados Literatura Latino-Americana e do Caribe em Ciências da Saúde (LILACS) e MEDLINE pela Pubmed, a partir da questão norteadora "Quais as evidências científicas sobre descolamento prematuro da placenta?". Resultados: predominaram estudos que não apresentam fortes evidências para aplicação clínica e a totalidade é de autoria médica. Os estudos analisados têm como foco a associação de DPP com patologias, alterações genéticas e comportamentais na gestação. Considerações finais: percebe-se a escassez com níveis de evidência altos, bem como, ausência de produções na área de enfermagem.

Descritores: Enfermagem obstétrica; Descolamento prematuro da placenta; Complicações na gravidez.
\end{abstract}

\section{ABSTRACT}

Objetive: this study aimed to analyze the scientific publications available in the literature on abruptio placentae. Methods: this is an integrative literature review. The search was conducted in the databases Latin American and Caribbean Health Sciences (LILACS) and MEDLINE by PubMed, from the guiding question "What are the scientific evidence of abruptio placentae?". Results: studies that do not show strong evidence for clinical application predominated, most medical authorship. The studies analysis were focused on the DPP association with diseases, genetic and behavioral changes during pregnancy. Final Considerations: there is a shortage with high levels of evidence, as well as the absence of productions in nursing.

Descriptors: Obstetric nursing; Abruptio placentae; Pregnancy complications.

\footnotetext{
1 Enfermeira Obstetra. Mestra em Saúde Materno Infantil. Centro Universitário Franciscano, Santa Maria, RS, Brasil. E-mail: brunadedavid.rocha@gmail.com

${ }^{2}$ Enfermeira Obstetra. Mestra em Enfermagem. Universidade Federal de Santa Maria, Santa Maria, RS, Brasil. Email: francislenelm@yahoo.com.br

${ }^{3}$ Enfermeira. Doutora em Enfermagem. Docente do Curso de Enfermagem e Mestrado em Saúde Materno Infantil, Centro Universitário Franciscano, Santa Maria, RS, Brasil. E-mail: claudiaz@unifra.br

4 Enfermeira Obstetra. Mestranda em Enfermagem pela Universidade Federal de Santa Maria. Professora Substituta no Departamento de Enfermagem da Universidade Federal de Santa Maria - Campus Palmeira das Missões. E-mail: irismessagomes@gmail.com

${ }^{5}$ Enfermeira Obstetra. Mestra em Saúde Materno Infantil. Centro Universitário Franciscano, Santa Maria, RS, Brasil. E-mail: jusbordignon@gmail.com
} 


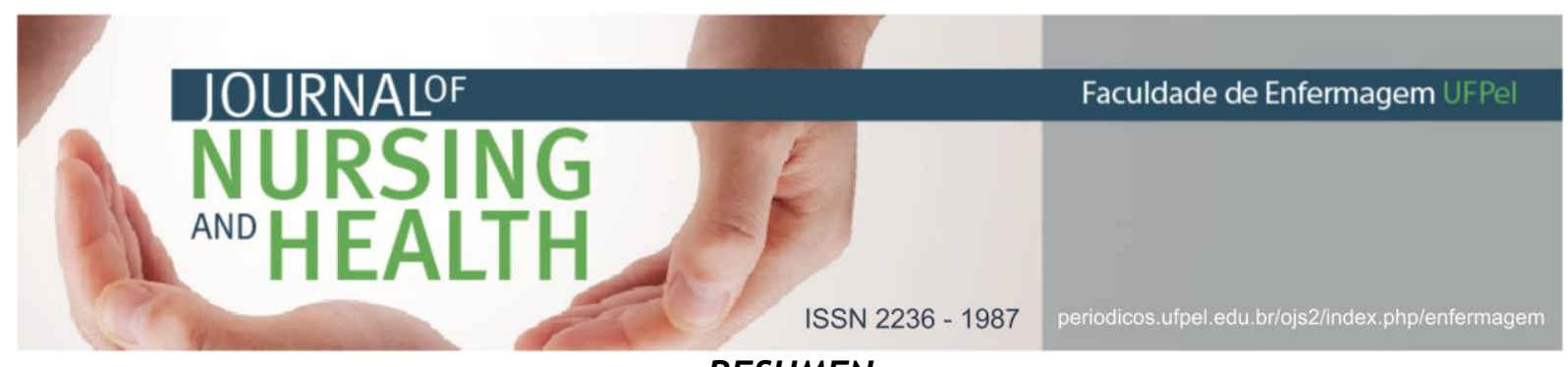

RESUMEN

Objetivo: analizar las publicaciones científicas disponibles en la literatura sobre el desprendimiento de la placenta. Métodos: se trata de una revisión integradora de la literatura. La búsqueda se realizó en las bases de datos (LILACS) de América Latina y el Caribe y Ciencias de la Salud MEDLINE de PubMed, de la pregunta guía "¿Lo que la evidencia científica de desprendimiento de la placenta?". Resultados: predominaron los estudios que no muestran una fuerte evidencia para la aplicación clínica y la mayoría son autoría médica. Los estudios analizados se han centrado en el análisis de la asociación DPP con enfermedades genéticas, y los cambios de comportamiento durante el embarazo. Consideraciones finales: hay una escasez con altos niveles de las pruebas, así como la ausencia de producciones en enfermería.

Descriptores: Enfermería obstétrica; Desprendimiento prematuro de la placenta; Complicaciones del embarazo.

\section{INTRODUÇÃO}

0 descolamento prematuro da placenta (DPP) é conceituado como a separação antecipada de parte ou da totalidade da placenta da parede do útero, que deveria se encontrar implantada até o nascimento do feto. Normalmente, essa intercorrência acontece no $3^{\circ}$ trimestre da gestação, embora possa ocorrer a qualquer momento, depois da $20^{a}$ semana de gravidez. O sangramento causado por DPP é classificado como hemorragia interna ou externa. Quando se localiza entre as membranas e o útero, sendo eliminado pelo colo, denomina-se hemorragia externa, que corresponde a $80 \%$ dos casos. Quando o sangue não é eliminado externamente, ficando retido entre a placenta descolada e o útero, recebe o nome de hemorragia oculta (20\% dos casos). ${ }^{1}$

O DPP é uma complicação obstétrica com taxa de incidência de $1 \%$ das gestações no intervalo de 24 a 26 semanas. Em relação às complicações fetais ocasionadas pela intercorrência, 10\% dos partos são prematuros, também podendo ocorrer óbito fetal (geralmente quando mais de $50 \%$ da placenta encontra-se com descolamento) e complicações importantes aos recém-nascidos que sobrevivem. Portanto, está associado com significativas taxas de morbimortalidade perinatal. ${ }^{2}$

Dentre os fatores de risco do DPP, cita-se a idade materna avançada, a multiparidade, hipertensão crônica, ruptura prematura de membranas, gestação múltipla, tabagismo e outras drogas, trombofilias, história de descolamento prematuro de placenta, fibroma uterino. ${ }^{3}$ Sendo que, o principal fator associado ao DPP é a hipertensão arterial, seja ela associada à préeclâmpsia ou à hipertensão crônica. ${ }^{1}$

$$
\text { o diagnóstico dessa }
$$
intercorrência é fundamentalmente clínico e manifestado por: dor repentina e intensa no abdome e/ou costas, contrações uterinas fortes e frequentes, hipertonia uterina, sangramento vaginal, hipertensão e padrão cardíaco fetal não tranquilizador. ${ }^{1}$ Também pode ser realizado um diagnóstico ultrassonográfico, visto que o quadro clínico pode confundir o profissional por existirem casos assintomáticos e sintomáticos. ${ }^{2}$ 
Em relação ao cuidado de enfermagem à gestante com DPP, o mesmo deve ser minucioso, pois exige uma avaliação meticulosa das condições materno-fetais. As informações do tratamento e suas causas devem ser concedidas para a mulher e para sua família e o apoio emocional também é excepcionalmente importante pela possibilidade de perda do feto e pela patologia crítica da mãe. ${ }^{2}$

Justifica-se a realização desse estudo, pela necessidade de os profissionais da saúde, principalmente médicos e enfermeiros, identificarem precocemente, fatores de risco para o descolamento prematuro da placenta, bem como, traçar estratégias para prevenir patologias e comportamentos que conduzam para esse desfecho, contribuindo para a redução das taxas de morbimortalidade materno-fetal. Dessa maneira, este estudo tem por objetivo analisar as produções científicas disponíveis na literatura sobre descolamento prematuro da placenta.

\section{MATERIAIS E MÉTODOS}

Para alcançar o objetivo do estudo, optou-se pelo método de revisão integrativa da literatura que tem por objetivo analisar e sintetizar os resultados encontrados, por meio de uma estratégia criteriosa para extrair evidências científicas que possam nortear as condutas na saúde. ${ }^{4}$ Neste estudo, não ocorreu o envolvimento direto de seres humanos, tendo em vista que, configura-se como uma revisão integrativa de literatura. Por esse motivo, não houve a necessidade de aprovação por um Comitê de Ética em Pesquisas com Seres Humanos. No entanto, as informações passaram pela revisão por pares, para garantir o rigor científico exigido nesse tipo de estudo.

Para a elaboração desta revisão integrativa, foram elencadas seis etapas: formulação da questão de pesquisa, delimitação de critérios de inclusão e exclusão para seleção dos estudos, coleta de dados, análise dos estudos elencados, interpretação dos resultados obtidos e apresentação da revisão. ${ }^{4}$ Para nortear a pesquisa, foi delimitada a seguinte questão norteadora: "Quais as evidências científicas sobre descolamento prematuro da placenta?".

A busca de produções científicas foi realizada em agosto de 2016, nas bases de dados eletrônicas Literatura Latino-Americana e do Caribe em Ciências da Saúde (LILACS) e MEDLINE pela Pubmed. A escolha pela busca nas referidas bases de dados se justifica, por contemplarem a literatura produzida na América latina e no Caribe, e abranger também produções internacionais na área da saúde.

Foram elencados como critérios de inclusão: publicações disponíveis na íntegra, em formato de artigo, nos idiomas português, inglês ou espanhol, de periódicos nacionais ou internacionais, com resumos disponíveis on-line, indexados nas bases de dados. Foi estabelecido um recorte temporal de cinco anos, entre 2011 e 2016. Foram excluídas do estudo: publicações que não abordassem sobre o descolamento prematuro da placenta, que não apresentassem resumo nas bases de dados e que estivessem em formato de 
teses, dissertações, documentos ministeriais, protocolos e livros.

$\mathrm{Na}$ base de dados LILACS, foi utilizado o descritor "descolamento prematuro de placenta", contemplado nos Descritores em Ciências da Saúde (DECS), por formulário iAH. A busca retornou 88 publicações. Após a leitura dos títulos e dos resumos dos artigos, 45 não estavam disponíveis online, 20 não contemplavam o tema da pergunta de pesquisa e sete não estavam em formato de artigo, 15 não haviam sido publicados nos últimos cinco anos, sendo selecionado apenas um artigo nesta base de dados.

$\mathrm{Na}$ base de dados MEDLINE pela PUBMED, foram utilizados os descritores em inglês do "Mesh Database", o operador booleano AND e delimitada a data de publicação, em formulário avançado: "abruptio placentae" AND "pregnancy complications" AND “2011 a 2016". A busca retornou 111 resultados e após leitura prévia do título e do resumo, foram selecionadas oito publicações, sendo que 69 não contemplavam especificamente o tema da pergunta de pesquisa e 34 não estavam disponíveis online. Após leitura integral dos artigos, foram selecionadas 6 publicações.

A amostra final foi composta de sete estudos. A coleta de informações deu-se por meio de um quadro sinóptico (Quadro 1) elaborado pelas pesquisadoras e aplicado para cada artigo selecionado. 0 quadro apresentou as seguintes informações: autor(es), periódico, ano de publicação, método e nível de evidência do estudo.
O nível de evidência de cada estudo foi atribuído com base em uma classificação com sete níveis, quais sejam: nível 1) evidências provenientes de revisões sistemáticas ou metanálises; nível 2) evidências derivadas de ao menos um ensaio clínico randomizado controlado bem delineado; nível 3) evidências de ensaios clínicos bem delineados sem randomização; nível 4) evidências provenientes de estudos de coorte e de caso-controle; nível 5) evidências de revisões sistemáticas de estudos descritivos e qualitativos; nível 6) evidências derivadas de um único estudo descritivo ou qualitativo; nível 7) evidências oriundas de opinião de autoridades e/ou relatórios de comitês de especialistas. ${ }^{5}$ A apresentação dos resultados e a discussão geral sobre o descolamento prematuro da placenta foi realizada de forma descritiva.

\section{RESULTADOS}

Pela seleção das publicações, constituíram o corpus de análise, o total de sete e-12 $^{6}$ artigos, sendo um do LILACS $^{6}$ e seis da PUBMED. ${ }^{7-12}$ Os artigos foram publicados em periódicos variados, um ${ }^{6}$ nacional e seis ${ }^{7-12}$ internacionais, todos de autoria médica. Em relação ao ano de publicação, a maioria dos artigos é dos anos de $2014^{7-8}$ (dois) e 20159-10 (dois).

Referente ao delineamento metodológico, predominam estudos clínicos randomizados do tipo casocontrole três ${ }^{9-10,12}$; um estudo descritivo transversal retrospectivo ${ }^{6}$; uma revisão sistemática com metanálise ${ }^{8}$; um estudo documental longitudinal ${ }^{11}$ e um relato de caso. ${ }^{7}$ 


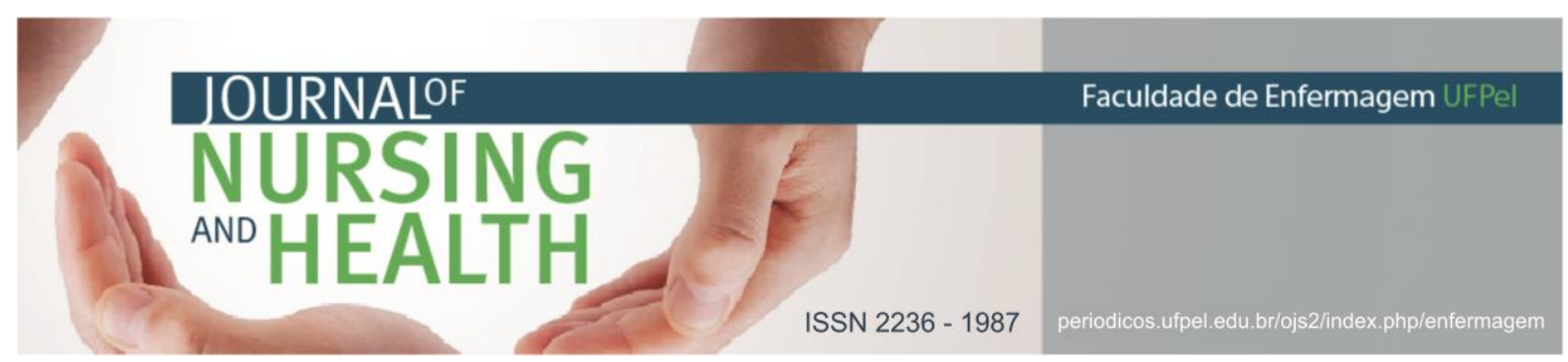

\begin{tabular}{|c|c|c|c|c|}
\hline AUTORES & PERIÓDICO & ANO & TIPO DE ESTUDO & $\begin{array}{l}\text { NÍVEL DE } \\
\text { EVIDÊNCIA }\end{array}$ \\
\hline Oliveira LC; Costa AAR. ${ }^{6}$ & $\begin{array}{l}\text { Revista da } \\
\text { Associação Médica } \\
\text { Brasileira }\end{array}$ & 2013 & $\begin{array}{l}\text { Estudo descritivo, } \\
\text { transversal, } \\
\text { retrospectivo }\end{array}$ & 2 \\
\hline $\begin{array}{l}\text { Honda M; Matsunaga A; Era } \\
\text { S; Takai Y; Baba K; Seki H. }\end{array}$ & $\begin{array}{l}\text { Journal of Medical } \\
\text { Case reports }\end{array}$ & 2014 & Relato de caso & 6 \\
\hline $\begin{array}{l}\text { Huang QT; Chen JH; Zhong } \\
\text { M; Xu YY; Cai CX; Wei SS; } \\
\text { Hong LL; Liu Q; Yu YH. }{ }^{8}\end{array}$ & Elsevier & 2014 & $\begin{array}{l}\text { Revisão } \\
\text { sistemática com } \\
\text { metanálise }\end{array}$ & 1 \\
\hline $\begin{array}{l}\text { Qiu C; Sanchez SE; Hevner K; } \\
\text { Enquobahrie DA; Williams } \\
\text { MA. }{ }^{9}\end{array}$ & $\begin{array}{l}\text { BMC Research } \\
\text { Notes }\end{array}$ & 2015 & $\begin{array}{l}\text { Estudo clínico } \\
\text { randomizado do } \\
\text { tipo caso- } \\
\text { controle }\end{array}$ & 4 \\
\hline $\begin{array}{l}\text { Qiu C; Sanchez SE; Gelayde } \\
\text { B; Enquobahrie DA; Ananth } \\
\text { CV; Williams MA. }{ }^{10}\end{array}$ & $\begin{array}{l}\text { The Journal of } \\
\text { Maternal-Fetal and } \\
\text { Neonatal Medicine }\end{array}$ & 2015 & $\begin{array}{l}\text { Estudo clínico } \\
\text { randomizado, } \\
\text { multicêntrico, do } \\
\text { tipo caso- } \\
\text { controle }\end{array}$ & 4 \\
\hline $\begin{array}{l}\text { Salihu HM; Diamond E; } \\
\text { August EM; Rahman S; Mogos } \\
\text { MF; Mbah AK.11 }\end{array}$ & Nutrition Reviews & 2016 & $\begin{array}{l}\text { Estudo } \\
\text { longitudinal, } \\
\text { documental }\end{array}$ & 6 \\
\hline $\begin{array}{l}\text { Moore A; Enquobahrie DA; } \\
\text { Sanchez SE; Ananth CV; } \\
\text { Pacora PN; Williams MA. }{ }^{12}\end{array}$ & $\begin{array}{l}\text { International } \\
\text { Journal of } \\
\text { Molecular } \\
\text { Epidemiology and } \\
\text { Genetics }\end{array}$ & 2012 & $\begin{array}{l}\text { Estudo clínico } \\
\text { randomizado do } \\
\text { tipo caso- } \\
\text { controle }\end{array}$ & 4 \\
\hline
\end{tabular}

Quadro 1 - Principais dados dos estudos selecionados. Santa Maria - RS. 2016

0 nível de evidência de cada estudo foi classificado em $1^{8}, 2^{6}, 4^{9-10,12}$ e $6^{7,11}$, com predomínio de nível 4 , os quais possuem mínimas evidências científicas satisfatórias, ou seja, consideradas insuficientes, contra ou a favor de uma recomendação os estudos, em sua maioria, são clínicos e objetivam analisar a associação de DPP a outros fatores, além dos mais comuns citados pela literatura. ${ }^{7-8,10-11}$ Destacam-se pesquisas que analisaram a associação com patologias, alterações genéticas, fisiopatológicas 


\section{JOURNALOF \\ NURSING \\ ANO HEALTH}

ISSN 2236 - 1987

e alterações comportamentais na gestação. 6,8-9,12

Dentre os estudos selecionados para essa revisão, o único estudo brasileiro analisou a relação de fatores maternos e neonatais e a ocorrência de near miss materno. Em uma amostra de 255 pacientes que estiveram internadas em UTI materna, $29(11,4 \%)$ dos casos foram em decorrência de DPP, sendo que $73,1 \%$ dos óbitos fetais foram causados por essa intercorrência. Esses dados demonstram que o DPP é identificado como um dos principais fatores desencadeadores de near miss materno, associado a óbito fetal e neonatal. ${ }^{6}$

Em relato de caso de gestante com DPP, os principais fatores de risco para sua ocorrência foram idade materna avançada e multiparidade. Em relação ao histórico gestacional, a paciente não apresentou DPP em partos anteriores, hipertensão arterial sistêmica ou tromboembolismo. Sobre as principais queixas, destacam-se a dor abdominal forte, contrações frequentes e útero hipertônico, sem presença de perdas vaginais. ${ }^{7}$

Dos estudos clínicos, uma publicação merece destaque por tratar-se de metanálise, sendo considerada o mais alto nível de evidência científica, com aplicabilidade para a prática clínica. No que se refere à associação de patologias prévias com 0 risco aumentado de desenvolver complicações placentárias, como DPP e placenta prévia, a metanálise conclui que não existe associação de hepatite B como aumento de risco para desenvolvimento dessas intercorrências. Porém, o estudo sinaliza a necessidade de pesquisas com metodologias mais criteriosas, para uma comprovação real ou não dessa associação. ${ }^{8}$

Em um dos estudos clínicos randomizados, do tipo caso-controle, os autores objetivaram avaliar a associação de alterações no número de cópias do DNA mitocondrial (mtDNA), no sangue materno, com a ocorrência de DPP em gestantes. Para tanto, foram avaliadas placentas após o parto e realizada análise do mtDNA. Os resultados demonstraram que, quanto maior o número de cópias mtDNA nas placentas, provenientes do sangue materno, maior a chance de ocorrência de DPP. ${ }^{9}$

Nessa mesma vertente, estudo realizado em Lima, no Peru, objetivou analisar a associação de variações nos genes maternos cardiometabólicos com o risco de DPP. Foram analisados polimorfismos de um único nucleotídeo (SNPs), ou seja, a sequência pontual de um DNA. No gene analisado, foram observadas associações com genes envolvidos na coagulação, reninaangiotensina, angiogênese inflamação e o metabolismo de vitamina $B$, com o risco de DPP. Além disso, o estudo traz que os casos de DPP estão diretamente relacionados com história materna pregressa de pré-eclâmpsia e eclâmpsia, DPP e falta de assistência pré-natal. $^{12}$

No que se refere às complicações materno-fetais após o DPP, no relato de caso analisado, a paciente sem histórico de complicações, apresentou coagulação intravascular disseminada (CIVD) com necessidade de transfusão sanguínea durante o trabalho de parto. 


\section{JOURNALOF \\ NURSING \\ M०HEALTH}

Após o parto, foi identificado hematoma retroplacentário, na avaliação placentária. ${ }^{7}$

Outro estudo clínico, realizado no Peru, analisou a associação de DPP com a duração do sono das gestantes e queixas de exaustão vital no início da gestação. Em gestantes que relataram curta duração de sono (menor ou igual a 6 horas diárias), as chances de ocorrência de DPP aumentaram em 1,90 vezes; Para gestantes que relataram longa duração de sono (maior ou igual a 9 horas diárias), as chances aumentaram em 1,98 vezes. Em relação à queixa de exaustão vital, a queixa aumentou em 2,4 vezes a chance de ocorrer DPP. Portanto, o estudo conclui que alterações metabólicas relacionadas à distúrbios do sono e estado mental como a exaustão vital, no início da gestação, podem ser considerados fatores de risco para complicações como a DPP e devem ser considerados pelos profissionais de saúde. ${ }^{10}$

Sobre o ganho de peso na gestação e sua associação com o risco de DPP, foi realizado um estudo no Missouri, com uma amostra de 1175.623 nascimentos entre os anos de 1989 e 2005. As recomendações sobre o peso foram baseadas na publicação do Instituto de Medicina, intitulada " $O$ ganho de peso na gravidez: reexaminando as orientações". De acordo com os resultados, $57,52 \%$ das pacientes obtiveram um ganho de peso ideal durante a gestação; $11 \%$ obtiveram um ganho de peso abaixo do ideal; $17,93 \%$ estavam acima do peso ideal e $13,54 \%$ eram obesas. 0 estudo concluiu que pacientes que tiveram um ganho de peso abaixo do ideal,
ISSN 2236 - 1987

perfaziam as taxas mais elevadas de ocorrências de DPP, enquanto que, pacientes com ganho de peso acima do ideal e as com excesso de peso tinham as menores taxas de DPP. Além disso, uma característica importante é que as pacientes com ganho de peso abaixo do ideal tinham idade materna avançada, considerada um fator de risco importante. ${ }^{11}$

No que concerne à via de parto, a literatura não possui um consenso, mas a cesariana é o procedimento mais realizado nesses casos, por se tratar de uma emergência obstétrica de alto risco materno-fetal. Em relato de caso sobre gestante japonesa, com 40 semanas de gestação, colo uterino favorável, sinais vitais maternos estáveis e ausência de batimentos cardiofetais, optou-se pelo parto vaginal induzido com ocitocina, para evitar uma intervenção mais invasiva. ${ }^{7}$

\section{DISCUSSÃO}

As complicações obstétricas relacionadas às intercorrências ou intervenções durante o trabalho de parto e parto podem evoluir para morbidades maternas como o near miss. Desordens hemorrágicas, como DPP, podem influenciar na evolução do quadro, portanto, devem ser observadas com atenção pelos profissionais da saúde. ${ }^{13}$

Corroborando com os estudos analisados, publicações atuais confirmam que os principais sintomas do DPP na prática clínica são: dor abdominal forte, hipertonia uterina e sangramento vaginal. Em dois relatos de caso, as pacientes não apresentaram perdas vaginais na admissão. Porém, em um dos estudos 


\section{JOURNALOF \\ AND}

NURSING

\section{ocorreu}

hemorragia puerperal importante, com necessidade de transfusão sanguínea e internação na Unidade de Terapia Intensiva (UTI). No segundo estudo, não foram relatadas complicações maternas, mas foi identificada a presença de hematoma retroplacentário, ou seja, coágulo formado por hemorragia retida. ${ }^{14-15}$

Dentre os fatores de risco para DPP, estudo de coorte realizado na Tanzânia apontou hipertensão crônica, pré-eclâmpsia/eclampsia, cesariana em parto anterior, DPP em parto anterior, poucas consultas de pré-natal e multiparidade. Como complicações maternas, ocorreram maiores taxas de hemorragia pré e pós-parto, cesariana, necessidade de transfusão sanguínea, função alterada do fígado e mortalidade materna. Para o recémnascido, destaca-se baixo peso ao nascer, prematuridade, índices de apgar abaixo de 7 no $1^{\circ}$ e $5^{\circ}$ minutos e óbito perinatal. ${ }^{16}$

As alterações placentárias estão diretamente relacionadas a distúrbios hipertensivos na gestação, como a préeclâmpsia ou hipertensão crônica, sendo as principais causas de DPP. ${ }^{3,17}$ Os distúrbios hipertensivos alteram a remodelação das artérias uteroplacentárias, as quais são responsáveis pela circulação uteroplacentária. ${ }^{18} \mathrm{Em}$ relato de caso ocorrido em hospital de Minas Gerais, a gestante apresentou aumento da pressão arterial, alterações visuais, dor abdominal forte e proteinúria de fita positiva na admissão do hospital, sendo diagnosticada com DPP, ocasionado por pré-eclâmpsia. ${ }^{19}$

Destaca-se que o DPP está entre uma das principais causas de
ISSN 2236 - 1987

hemorragia puerperal e de coagulopatias maternas. Para tanto, é necessária uma avaliação rigorosa no puerpério, nos casos de intercorrências como essa. Os profissionais devem estar atentos para os exames laboratoriais tais como contagem de plaquetas e tempo de protrombina, para controle do estado de coagulação da paciente. Também é importante prever a necessidade de transfusão de componentes sanguíneos e promover sua instalação adequada. ${ }^{20}$

Sobre a via de parto, a literatura, em sua maioria, traz que a cesariana de urgência é a conduta mais realizada e viável nos casos de DPP. Porém, em um dos estudos, dos 318 casos de DPP que ocorreram em um hospital escola estrangeiro, $70 \%$ tiveram como desfecho o parto vaginal. ${ }^{17}$

Não foram encontrados estudos recentes sobre associação de DPP com hepatite $B$, distúrbios do sono e exaustão vital, DNA mitocondrial e SNPs, visto que, trata-se de um tema com escassez de publicações, principalmente, nacionais e de autoria de enfermeiros.

Em relação à prática profissional em casos de DPP, não foram encontrados, na literatura, artigos acerca dessa questão para nortear a discussão. Principalmente por serem de autoria médica, as publicações são focadas na questão da conduta do profissional médico e no manejo clínico dessa intercorrência. Porém, destaca-se que o trabalho em equipe merece ser destacado e abordado nos artigos, visto que, a decisão clínica deve ser tomada em conjunto com todos os membros. Salienta-se a necessidade de mais estudos sobre 


\section{JOURNALOF \\ NURSING \\ aNO HEALTH}

ISSN 2236 - 1987

intercorrências

obstétricas

que envolvam a equipe de saúde e de protocolos baseados em evidências, sobre a assistência que deve ser prestada nesses casos, por enfermeiros, médicos e demais profissionais da área.

\section{CONSIDERAÇÕES FINAIS}

Ao analisar os estudos selecionados para a realização dessa revisão, percebe-se a escassez de produções relacionadas à temática e que sejam baseadas em evidências científicas, que possam nortear a prática dos profissionais da saúde. Também é importante destacar que, em sua totalidade, as produções são de autoria médica e são voltadas principalmente para aspectos clínicos relacionados à DPP, sendo que a assistência como um todo não é discutida.

Portanto, destaca-se a necessidade de produções científicas sobre intercorrências obstétricas, tais como o DPP, por profissionais da saúde, principalmente por enfermeiros, com enfoque na atuação multiprofissional, qualificada e humanizada nesses casos. Percebe-se uma discussão pontualmente clínica e voltada somente para a figura do médico, sendo que, é de suma importância envolver a equipe de saúde para que o cuidado seja realizado de maneira integral $e$ individualizada.

Essa revisão apresentou em sua maioria, publicações oriundas de estudos clínicos, que objetivaram analisar a associação de DPP com patologias específicas, comportamentos maternos durante a gestação e alterações fisiológicas e genéticas. A partir da análise, foram classificados com pouca relevância nos níveis de evidências. Sugere-se que novos estudos sejam realizados na área, com foco na qualidade da assistência às intercorrências obstétricas, por profissionais enfermeiros, no sentido de subsidiar a prática profissional.

\section{REFERÊNCIAS}

1. Freitas F, Martins-Costa $\mathrm{SH}$, Ramos JGL, Magalhães JA. Rotinas em obstetrícia. $6^{\mathrm{a}}$ ed. Porto Alegre. Artmed; 2011.

2. Montenegro CAB, Filho JR. Obstetrícia fundamental. $12^{\mathrm{a}}$ ed. Rio de Janeiro. Guanabara Koogan; 2013.

3. Cunningham FG, Leveno KJ, Bloom SL, Hauth JC, Rouse DJ, Spong CY. Obstetrícia de Williams. 23 ${ }^{\mathrm{a}}$ ed. Porto Alegre: AMGH; 2012.

4. Soares CB, Hoga LAK, Peduzzi M, Sangaleti C, Yonekura T, Silva DRAD. Revisão integrativa: conceitos e métodos utilizados na enfermagem. Rev esc enferm USP [Internet]. 2014 [acesso em 2016 ago 8];48(2):335-45. Disponível em: http://www.scielo.br/pdf/reeusp/v48 n2/pt_0080-6234-reeusp-48-02335.pdf

5. Melnyk BM, Fineout-Overholt E. Making the case for evidence-based practice. In: Melnyk BM, FineoutOverholt E, editors. Evidence-based practice in nursing and healthcare: a guide to best practice. $1^{\text {a }}$ ed. Philadelphia: Lippincout Williams and Wilkins, 2005. p. 3-24. 


\section{E \\ ISSN 2236 - 1987}

6. Oliveira LC, Costa AAR. Óbitos fetais e neonatais entre casos de near miss materno. Rev assoc med bras [Internet]. 2013 [acesso em 2016 ago 10];59(5):487-94. Disponível em: http://www.scielo.br/pdf/ramb/v59n 5/v59n5a14.pdf

7. Honda M, Matsunaga S, Era S, Takai Y, Baba K, Seki H. Intrapartun antidisseminated intravascular coagulation therapy leading to successful vaginal delivery following intrauterine fetal death caused by placental abruption: a case report. J med case rep [Internet]. 2014 [acesso em 2016 ago 10];8(461):1 5. Disponível em: http: / /www.ncbi.nlm.nih.gov/pmc/ar ticles/PMC4307188/

8. Huang QT, Chen JH, Zhong M, Xu YY, Cai CX, Wei SS, et al. The risk of placental abruption and placenta previa in pregnant women with chronic hepatitis B viral infection: a systematic review and meta-analilysys. Placenta [Internet]. 2014 [acesso em 2016 ago 10];35:539-45. Disponível em: http:/ / www.placentajournal.org/artic le/S0143-4004(14)00220-3/pdf

9. Qiu C, Sanchez SE, Hevner K, Enquobahrie DA, Williams MA. Placental mitochondrial DNA contente and placental abruption: a pilot study. BMC res notes [Internet]. 2015 [acesso em 2016 ago 10];8(447):1-6. Disponível em:

http:/ / bmcresnotes. biomedcentral.co m/articles/10.1186/s13104-015-13404

10. Qiu S, Sanchez SE, Gelayde B, Enquobahrie DA, Ananth CV, Williams MA. Maternal sleep duration and complaints of vital exhaustion during pregnancy is associated with placental abruption. J matern fetal med [Internet]. $2015 \mathrm{fev}$ [acesso em 2016 ago 10];28(3):350-5. Disponível em: http://www.tandfonline.com/doi/abs /10.3109/14767058.2014.916682?jour nalCode=ijmf20

11. Salihu HM, Diamond E, August EM, Rahman S, Mogos MF, Mbah AK. Maternal pregnancy weight gain and the risk of placental abruption. Nutr rev [Internet]. 2016 [acesso em 2016 ago 10];71 Suppl1:S9-17. Disponível em:

http://nutritionreviews.oxfordjournal s.org/content/71/suppl_1/S9.long

12. Moore A, Enquobahrie DA, Sanchez $\mathrm{SE}$, Ananth CV, Pacora PN, Williams MA. A genome-wide association study of variations in maternal cardiometabolic genes and risk of placental abruption. Int $\mathrm{j}$ mol epidemiol genet [Internet]. 2012 [acesso em 2016 ago 10];3(4):305-13. Disponível em: http://www.ncbi.nlm.nih.gov/pmc/ar ticles/PMC3508543/

13. Viana RC, Novaes MRCG, Calderon IMP. Mortalidade materna - uma abordagem atualizada. Comun ciênc saúde [Internet]. 2011 [acesso em 2016 ago 10];22 Suppl 1:141-52.Disponível em:

http: / / www.escs.edu.br/pesquisa/rev ista/2011Vol22_16mortabilidade.pdf

14. Vitória KM, Ennes LO, Tavares RF, Araújo ICOA, Zwetkoff BF, Reis AS, et al. Descolamento prematuro de placenta: relato de caso. Rev med Minas Gerais [Internet]. 2011 [acesso em 2016 ago 11];21(2 Suppl 4):S1-S113. Disponível em: http://www.rmmg.org/artigo/detalhe s/898 


\section{NURSING \\ ANO HEALTH}

15. Zullino S, Faiola S, Paganelli AM, Ferrazzi E. A case of abruptio placentae due to torsion of gravid uterus. Case rep obstet gynecol [Internet]. 2014 [acesso em 2016 ago 11];2014:1-3. Disponível em: http://www.hindawi.com/journals/cr iog/2014/801616/abs/

16. Macheku GS, Philemon RN, Oneko O, Mlay OS, Masenga G, Obure J, et al. Frequency, risk factors and fetomaternal outcomes of abruptio placentae in Northern Tanzania: a registry-based retrospective cohort study. BMC pregnancy childbirth [Internet]. 2015 [acesso em 2016 ago 10];15(1):1-10. Disponível em: https://bmcpregnancychildbirth.biom edcentral.com/articles/10.1186/s128 84-015-0678-x

17. Mukherjee S, Bawa AK, Gadam M. Retrospective study of risk factors and maternal and fetal outcome in patients with abruptio placentae. J nat sci biol med [Internet]. 2014 [acesso em 2016 ago 12];5(2)425-8. Disponível em: https://www.ncbi.nlm.nih.gov/pmc/a rticles/PMC4121928/

18. Villasmil ER, Pérez CB, Bolívar JS. Alteración de la placentación profunda en la preeclmapsia. Rev obstet ginecol venez [Internet]. 2012 [acesso em 2016 ago 10];72(4):269-76. Disponível em: http://www.scielo.org.ve/pdf/og/v72 n4/art08.pdf

19. Paiva SPC, Filho NJS, Parola AR. Útero de Couvelaire: relato de caso. Rev med Minas Gerais [Internet]. 2015 [acesso em 2016 ago 12];25(2):287-91. Disponível em: http://www.rmmg.org/artigo/detalhe s/1786
ISSN 2236 - 1987

20. Bonomi IBA, Cunha SM, Trigueiro MC, Lobato ACL, Tavares RP. Prevenção e manejo da hemorragia pós-parto. Rev med Minas Gerais [Internet]. 2012 [acesso em 2016 ago 12];22 Suppl 2:S1-S173. Disponível em: http://www.rmmg.org/artigo/detalhe s/715

Data de submissão: 28/07/2016

Data de aceite: 05/03/2017

Data de publicação: 30/08/2017 